\title{
340 - VISUAL HALLUCINATIONS SECONDARY TO ROPINIROLE: CASE REPORT
}

Authors: A.M. Carvalheiro, Joana Maia; Leiria Hospital Center, Psychiatry and Mental Health Service, Leiria, Portugal

Objectives: Using as a starting point a clinical case, the authors performed a literature review to clarify the relationship between visual hallucinations and treatment with ropinirole.

Methods: Analysis of the patient's clinical process and brief review of the latest available literature on the subject, published in PubMed/Medline databases.

Results: Female patient, 89 years old, without psychiatric illness, brought to the emergency room by visual hallucinations, in the past 3 days "I see red, blue and green spots and roses on your sweater and a lot of flowers on that lady's blouse"sic. She recognised them as unreal (pseudo hallucinations) "no, nothing is there. It's from my eyes. I am fine of the head"sic. She has a personal history of glaucoma for decades, and restless legs syndrome for about 1 year, medicated with ropinirole. Adherence to therapeutic has been explored and it was found that she has been increasing, progressively and by its own initiative, the dose of ropinirole. She claims to be currently taking two pills of $8 \mathrm{mg}$ twice daily (the recommended daily dose is $24 \mathrm{mg}$ ).

Conclusions: Studies indicate that the incidence of hallucinations during the treatment of RLS with ropinirole is less than $1 \%$, which can be justified by its high affinity for D3 receivers compared to D2 receivers. However, it is also known that the over-stimulation of dopamine receptors (by overdose or rapid titration) can cause hallucinations, which may have been the cause of the patient's clinical condition. This clinical case also allows to alert for the importance of excluding organic causes in the diagnosis of visual hallucinations.

Key words: Visual hallucinations; Restless Legs Syndrome; Ropinirole 